\title{
Propagation Characteristics and Channel Model of Short Wave Radio Waves at Sea
}

\author{
Guanzheng Zhang \\ North China Electric Power University, Baoding 071000, China; \\ 1194533191@qq.com
}

Keywords: Signal reflection, 3D modeling, Simulation analysis

\begin{abstract}
Aiming at the propagation characteristics of HF radio waves in the ionosphere and complex ocean environment, this paper introduces the modeling method and analysis method of HF radio propagation model on the sea from the aspect of signal fading. Firstly, a reflection model of ocean signal is established, in which the loss of electromagnetic wave propagation consists of three parts: free space loss, ionospheric loss and sea surface reflection loss. Secondly, the ship sloshing caused by ocean waves is modeled in three-dimensional. Then, the ocean signal reflection model is improved by considering the earth curvature and the antenna receiving gain caused by the antenna angle change. Finally, a marine signal reflection model suitable for ships to run on high seas is established. In this paper, a series of algorithms, such as simulation and analysis, are used to obtain a theoretical model of marine channel which is applicable to different maritime communication environments (different carrier frequencies, different communication distances, different ship sloshing forms and different sea conditions). Compared with the actual data, the model can better reflect the model.
\end{abstract}

\section{Introduction}

In order to study the problem of multi-hop short-wave radio propagation, we have established the model of short-wave radio propagation on the sea. The model of short-wave radio propagation on the sea is established mainly according to the transmitting frequency, elevation angle of the transmitting station and the reflection coefficient on the sea surface. The radius of curvature of the sphere is calculated and the appropriate results are obtained. Finally, the rationality of the model is verified.

\section{Organization of the Text}

\subsection{Ocean signal reflection model parameters.}

Due to the complex and changeable ocean climate, wave fluctuation and other factors, the radio wave propagation in the ocean environment is very complicated, resulting in changes in the parameters of the ocean signal reflection model. Before the signal reflection model is established, 
six important model parameters are introduced, which are sea surface RMS wave height, sea surface roughness, sea dielectric constant, Fresnel reflection coefficient and mirror reflection coefficient.

表 2 Douglas 海情级数

Table2 The sea condition level of Douglas

\begin{tabular}{|c|c|c|}
\hline \multirow{2}{*}{$\begin{array}{l}\text { 海情 } \\
\text { 级数 }\end{array}$} & \multicolumn{2}{|c|}{ Douglas } \\
\hline & 均方根波高 $(m)$ & 海面粗糙度描述 \\
\hline $\mathbf{O}$ & $\mathbf{o}$ & 平静的 \\
\hline $\mathbf{1}$ & $<0.3$ & 光滑的 (小浪) \\
\hline 2 & $0.3-0.9$ & 轻微的 (轻浪) \\
\hline 3 & $0.9-1.5$ & 缓和的 (中浪) \\
\hline 4 & $1.5-2.4$ & 粗糙的 (强浪) \\
\hline 5 & 2.4-3.7 & 很粗糙 (區浪) \\
\hline 6 & $3.7-6.1$ & (狂浪) \\
\hline 7 & $6.1-12.2$ & 很高的 (怒浪) \\
\hline 8 & 12.2 以上 & 陡峭的 (汹涛) \\
\hline 9 & $\cdots$ & $\ldots$ \\
\hline
\end{tabular}

\subsection{Attenuation model in free space and ionosphere.}

In order to calculate the attenuation of the wave in this process, the incident elevation angle is set at 50 degrees and the incident frequency is set at $10 \mathrm{MHz}$.

\subsubsection{Decay model in free space}

The loss of electromagnetic wave in air can be approximately determined by the energy attenuation model of electromagnetic wave in free space.

\subsubsection{Ionospheric decay model.}

When radio waves propagate in the ionosphere, they will encounter various obstacles and the influence of a large number of charged particles in the ionosphere. After reflection, scattering and diffraction, they will reach the receiver and become multi-path synthetic waves with small time difference. The propagation of radio waves in the ionosphere is affected by many factors. To simplify the discussion, we have made the following assumptions:

(1) Without considering the influence of geomagnetic field, the ionosphere is isotropic.

(2) Because the absorption of radio waves by $\mathrm{F}$ and $\mathrm{E}$ layers is usually very small, the effect of $\mathrm{D}$ layer absorption is only considered in this paper.

(3) There is symmetry in the propagation of electromagnetic waves in free space and ionosphere.

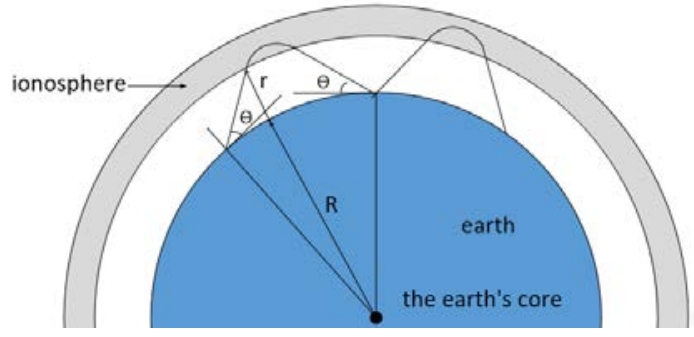

Fig.1 


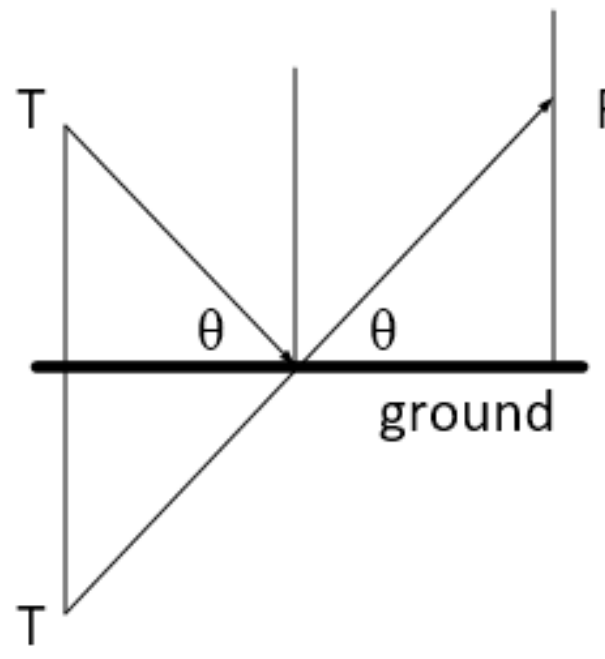

Reflection of smooth ground

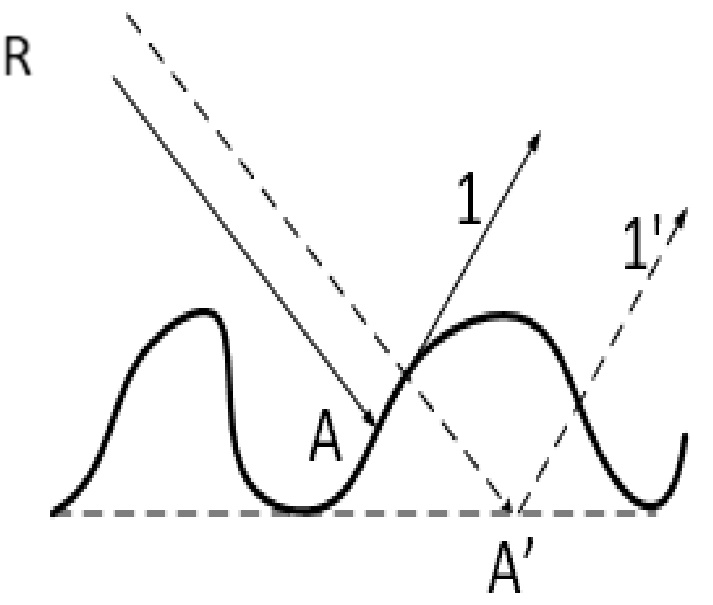

Reflection from rough ground

Fig.2

when reflecting on smooth surface, but when reflecting on rough surface, with the increase of roughness of the surface, the reflection coefficient decreases and the loss of energy increases gradually. This is basically consistent with our conclusions in the sea reflection model.

\section{Signal reflection model considering earth curvature and ship sloshing.}

\subsection{Reflection model of ship sloshing}

Surface waves are generally divided into three types: wind-induced waves, gravity and centrifugal forces that cause tides and tectonic tsunamis. This paper only considers the effects of wind waves.

Ships move on the waves as the waves move up, down, left and right. This can abstract the six degree of freedom motion model of ships ( $\mathrm{x}, \mathrm{y}, \mathrm{Z}$, alpha, beta, gamma).

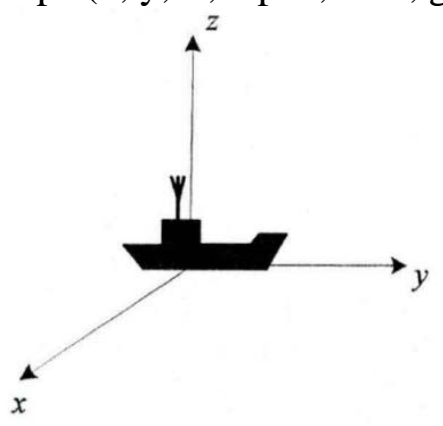

Fig.3

We establish a spherical coordinate system $(\mathrm{O}, \mathrm{x}, \mathrm{y}, \mathrm{z})$ for the earth's benchmark, and $\mathrm{O}$ is the center of the earth. The coordinates of other coordinate systems can also be transformed into coordinates of the coordinate system. So the ship's motion can be expressed as:

(1) The height changes along the $\mathrm{Z}$ axis.

(2) The left and right wobble is rotated at the center of the $X$ axis.

(3) The tilt of the front and rear axis revolves around the $Y$ axis. 


\subsection{Marine propagation model combined with ship sloshing.}

In the wave propagation model, the change of antenna's pitch and roll angle is combined with the change of antenna's gain in different directions. In this paper, the radiation vector parameter $G$ is used to represent the antenna gain and its component state in different directions.

\section{Summary}

In this paper, a series of algorithms, such as simulation and analysis, are used to obtain a theoretical model of marine channel which is applicable to different maritime communication environments (different carrier frequencies, different communication distances, different ship sloshing forms and different sea conditions). Compared with the actual data, the model can better reflect the model. Physical truth.

\section{References}

[1] Information on: www.cnki.com.cn

[2] Zhang Yu: Application Research of data mining technology in high risk personnel crime information mining, Master's degree, Nanchang University, China,2011 p.25

[3] Maojiali: Improvement of K-MEAN clustering based on genetic algorithm[D],Beijing University of Chemical Technology,China,2003 